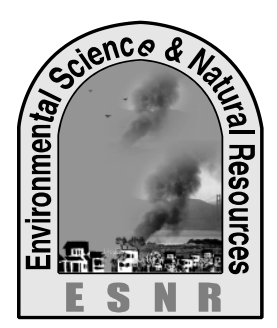

\title{
A Possible Approach for Maintaining Effective Omega-6/ Omega-3 Fatty Acid Ratio from Mixed Vegetable Oils
}

\author{
M. M. Haque ${ }^{1}$, M. N. Rahman ${ }^{2}$, M. J. Alam ${ }^{2 *}$ and S. Akter ${ }^{2}$ \\ ${ }^{1}$ Quality Control department, Igloo Ice-cream, Monem Group of Industries, Dhaka-1205, \\ Bangladesh. \\ ${ }^{2}$ Department of Food Technology and Nutritional Science, Mawlana Bhashani Science and \\ Technology University, Santosh, Tangail-1902, Bangladesh. \\ *Corresponding author: jahangirftns@gmail.com.
}

\begin{abstract}
Vegetable oil rich in omega-3 and omega- 6 fatty acids is an important element in the diet of most transitional countries. The ratio of omega-6 to omega-3 in modern diets is approximately $15: 1$, whereas ratios of $2: 1$ to $4: 1$ have been associated with reduced mortality from cardiovascular disease, suppressed inflammation in patients with rheumatoid arthritis, and decreased risk of breast cancer. The study was designed to investigate the fatty acid profile of six types of seed oils such as peanut, linseed, olive, soybean, sesame and sunflower oil. Afterwards the author prepared mixed vegetable oils with effective Omega-6 (n-6)/omega-3 (n-3) fatty acid ratio. It was found that the highest percentage $(39.9 \%)$ of saturated fatty acid found in Linseed oil and the highest percentage (37.1\%) of monounsaturated fatty acid found in Sesame oil. It was also observed that olive and soybean oil contain $100 \%$ polyunsaturated fatty acid and the lowest percentages $(35.2 \%)$ of polyunsaturated fatty acid were found in Sesame oil. After preparing a mixed vegetable oil The ratio of n-6 to $n-3$ were 3.5:1 (soybean), 19:1 (olive), 0.43:1 (linseed), 0.13:1 (peanut) and sesame (16.5:1). It is also noted that $n-3$ was not detected in sunflower oil. Thus the investigation showed that Soybean oil contains the balanced omega-6/omega-3 fatty acid ratio than others.
\end{abstract}

Key words: Cardiovascular disease, Edible oil, Essential fatty acids and Modern diet

\section{Introduction}

Dietary fats naturally present in food and play an important role in nutrition. It supply energy, carries fatsoluble vitamins (A, D, E and K) and is a source of antioxidants and bioactive compounds. Fats are also incorporated as structural components of the brain and cell membranes. They also play an important role in food preparation by enhancing food flavor, adding mouth-feel, making baked products tender, and conducting heat during cooking (USDA, 1997). Fatty acids is a carboxylic acid consisting of a hydrocarbon chain and a terminal carboxyl group, especially any of those occurring as esters in fats and oils. Saturated fatty acids do not contain any double bonds or other functional groups along the chain. If there is one or more double bonds in the fatty acid, it is no longer considered saturated, but rather, mono- or

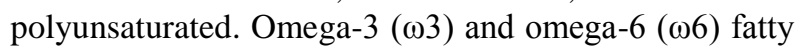
acids are unsaturated "Essential Fatty Acids" (EFAs) that need to be included in the diet because the human metabolism cannot create them from other fatty acids (Wijendran and Hayes, 2004). Since these fatty acids are polyunsaturated, the terms n-3 PUFAs and n-6 PUFAs are applied to omega- 3 and omega- 6 fatty acids, respectively. Omega- 6 fatty acids found in vegetable oils, nuts and seeds are a beneficial part of a hearthealthy eating plan. Omega-6 and omega-3 PUFA play a crucial role in heart and brain function and in normal growth and development (Binkoski et al., 2005). Linoleic and arachidonic acid are omega- 6 fatty acid that plays an important role in lowering cholesterol levels. Alpha linolenic and Docosahexaenoic acid are Omega-3 fatty acids can have health benefits when consumed in the recommended amounts, especially when used to replace saturated fats or trans fats in the diet. A meta-analysis of several trials indicated that replacing saturated fats with PUFA lowered risk for heart disease events by 24 percent (Bucher et al., 2002). When saturated fat in the diet is replaced by omega- 6 PUFA, the blood cholesterol levels go down (Harris et al., 2007). Omega-3 exists in three forms Alphalinolenic acid (found in vegetable sources such as peanut, linseed and soybean oil), Eicosapentaenoic and Docosahexaenoic acid (found in primarily in cold-water fatty fish such as salmon mackerel, lake trout, herring, sardines etc.). Omega-6 also exists in several forms. The first is linoleic acid which is found in olive oil, linseed oil linseed oil etc. Two other forms of omega- 6 are GLA (gamma-linolenic acid) and ARA (arachidonic acid). GLA is also found in plant based oils. ARA is found in many animal based foods.

Both $n-3$ and $n-6$ fatty acids are essential, i.e. humans must consume them in the diet. $n-3$ and $n-6$ compete for the same metabolic enzymes, thus the $n-6: n-3$ ratio will significantly influence the ratio of the ensuing eicosanoids (hormones), (e.g. prostaglandins, leukotrienes, thromboxanes etc.), and will alter the body's metabolic function (Tribole et al., 2006). Metabolites of $n-6$ are significantly more inflammatory (esp. arachidonic acid) than those of $n-3$ (Okuyama, 2001). This necessitates that $n-3$ and $n-6$ be consumed in a balanced proportion; healthy ratios of $n-6: n-3$ range from $1: 1$ to $4: 1$. The ratio of omega- 6 to omega-3 in modern diets is approximately $15: 1$, whereas ratios of $2: 1$ to $4: 1$ have been associated with reduced mortality from cardiovascular disease, suppressed inflammation in patients with rheumatoid arthritis, and decreased risk of breast cancer. Riediger et al. (2009) suggested that health benefits may be achieved by lowering dietary n6: n-3 FA even in a high fat diet. Riediger et al. (2009) demonstrated that lowering dietary ratio of $n-6: n-3$ fatty acids may significantly reduce cardiovascular and metabolic risks in mice regardless of the source of n-3 fatty acids (Simopoulos, 2008). In the secondary 
prevention of cardiovascular disease, a ratio of 4/1 was associated with a $70 \%$ decrease in total mortality. The lower omega-6/omega-3 ratio in women with breast cancer was associated with decreased risk. A ratio of 2$3 / 1$ suppressed inflammation in patients with rheumatoid arthritis, and a ratio of 5/1 had a beneficial effect on patients with asthma, whereas a ratio of 10/1 had adverse consequences. In the context of Bangladesh vegetable oils which are consumed by peoples do not contain actual ratio of $n-6 / n-3$ fatty acid. In this perspective, the author wanted to elucidate the fatty acid profile of six types of seed oils. Afterwards the author prepared mixed vegetable oils with effective n6/n-3 fatty acid ratio.

\section{Materials and Methods}

\section{Reagents used in the analytical process}

All chemicals, biochemical standards, reagents and solvents were of analytical grade purchased from Dhaka, Bangladesh.

\section{Collection of fats and oils}

Six different sample such as soybean oil, sunflower oil, sesame oil, peanut oil, linseed oil and olive oil were used in this study which were collected from different parts of Bangladesh. All samples were preserved in dry, brown bottles. The plastic bottles were covered with carbon papers to prevent photo oxidation. The bottles were also stored at $-28^{0} \mathrm{C}$ until analysis to prevent auto oxidation.

\section{Determination of fatty acid composition of fats and oils}

Before determining the fatty acid composition of lipid by Gas Liquid Chromatography (GLC) the fatty acids were converted to their methyl esters. The procedure followed was essentially the same as described in details by Yusuf et al. (1993).

\section{Preparation of fatty acid methyl esters}

\section{Preparation of ethanolic potassium hydroxide}

Potassium hydroxide (11.2g) was dissolved in $100 \mathrm{ml}$ of $95 \%$ ethyl alcohol and the solution was filtered.

\section{Preparation of anhydrous methanolic $\mathrm{HCl}(5 \% \mathrm{w} / \mathrm{w})$}

Traces of water were removed from methanol by allowing it to stand overnight in contact with fused anhydrous coarse powdered calcium chloride and then either filtering or centrifuging the suspension. The dry methanol thus obtained was stored in a tightly Stopper brown bottle. Methanol $\mathrm{HCl}$ mixture was prepared in the following manner. Hydrochloric acid gas also dry was generated from $\mathrm{NaCl}$ contained in a quick fit round bottom flask, with concentrated $\mathrm{H}_{2} \mathrm{SO}_{4}$ added to the salt drop by drop from a separating funnel/burette. The gas produced was passed through a quick-fit conical flask filled with the fused $\mathrm{CaCl}_{2}$ powder. The gas was introduced into a known amount of dry methanol contained in another bottle. The gas was passed until it was at least $5 \%(\mathrm{w} / \mathrm{w})$ in solvent (methanol). Usually a $7 \% \mathrm{HCl}$ in methanol mixture was prepared. Traces of $\mathrm{CaCl}_{2}$ powder were found to sediment on standing and were removed by filtration. This $\mathrm{HCl}$ : methanol reagent was stored in a tightly stopper bottle.

\section{Methylation of fatty acids}

Total lipid (400-600 mg) was taken in a ground joint flask and saponified with $15-30 \mathrm{ml} 2 \mathrm{M} \mathrm{KOH}$ (ethanolic) in water bath at $70^{\circ} \mathrm{C}$ for 1 hour by joining with a condenser. After cooling, the solution was diluted with equal volume of distilled water and acidified with concentrated $\mathrm{HCl}$ to $\mathrm{P}^{\mathrm{H}}<2$ as ascertained with a $\mathrm{P}^{\mathrm{H}}$ meter. The liberated fatty acids (a mixture) were extracted with $30-60 \mathrm{ml}$ of diethyl ether. Small amount of water was also extracted along with free fatty acids. This undesired water was removed by adding anhydrous sodium sulphate. The ether extract devoid of water was collected in another joint flask/filtration. The extract was then evaporated to dryness under $\mathrm{N}_{2}$. Dry methanolic $\mathrm{HCl}(25-50 \mathrm{ml})$ prepared as above, was added into the flask containing the fatty acid mixture and the content was heated at $85^{\circ} \mathrm{C}$ under reflux for 2 hours. After cooling, the fatty acids methyl esters (FAME) were extracted three times with equal volume of petroleum spirit $\left(\mathrm{bp} 40-60^{\circ}\right)$. All extracts were combined and evaporated to a small volume under $\mathrm{N}_{2}$.

\section{Purification of fatty acid methyl esters (FAME)}

FAME prepared as above was accompanied by free fatty acids, free cholesterol, cholesterol esters and other solvent impurities. Before analysis by GLC, they were purified by Thin-Layer Chromatography (TLC). A slurry of silica gel for thin layer chromatography is made with water $(2 \mathrm{ml}$ water per gm silica gel $\mathrm{G})$ in a beaker $(500 \mathrm{ml}$ capacity) and spread on $2 \mathrm{~mm}$ thick glass plates $20 \times 20 \mathrm{~cm}$ by a TLC spreader. The silica gel coating is $250 \mu \mathrm{m}$. The slurry thus spread is kept on the platform for about 10 minutes, transfer to the metal racks and dried in an oven at $110^{\circ} \mathrm{C}$ for about an hour. The plates are now ready for use.

\section{Thin Layer Chromatographic (TLC) procedure}

Standard fatty acids preparation $(\sim 3-5 \mathrm{ml})$ is now spotted on the plates with a glass capillary taking precaution so that not more than $2-3 \mu \mathrm{l}$ are spotted on the plates at a distance nearly $3 / 4$ for an inch from one edge on the plates. The gaps between two spots should be around half an inch and the spots should be as small as possible for better resolution of the fatty acids. The unknown should be spotted on the two locations. After air drying the plate is dipped in the solvents ( $\mathrm{n}$ - hexane: Diethyl ether: glacial acetic acid 70:30:1) in the TLC jar which is pre-equilibrated with the solvent system for about an hour. The solvent rise up the silica gel ( ascending chromatography) and is allowed to rise approximately anywhere between $15-18 \mathrm{~cm}$ ( nearly one hours) at which point the plate is removed from the jar, air dried, placed in the iodine chamber for 5 minutes. The FAME band in the plate was visualized in the iodine chamber. The FAME in the sample can be identified by their $\mathrm{R}_{\mathrm{f}}$ values when compared to standard. After the yellow color vanished the band was scraped into a centrifuge tube and eluted with methanol. The 
tube was then centrifuged and the supernatant was transferred into a dry flask. The FAME solution was evaporated to dryness under nitrogen. A small volume of dichloromethane solution was added to re-dissolve the FAME band and a 5-10 micro liter aliquot was analyzed in Gas-liquid chromatography.

Distance traveled by particular fatty acid

$\mathrm{R}_{\mathrm{f}}$

Distance traveled by the solvent front

Gas-Liquid Chromatographic (GLC) analysis of fatty acid methyl esters

The fatty acid methyl esters, prepared and purified as above, were analyzed by gas-liquid chromatography (GLC). A $2 \times 4 \mathrm{~mm}$ inside diameter column (Preferably glass) packed with $12-15 \%(\mathrm{w} / \mathrm{w})$ ethylene glycol succinate liquid phase coated on 100/200 mesh Gaschrom $\mathrm{P}$ was used. The injector temperature was $190^{\circ} \mathrm{C}$ and the detector temperature was $260^{\circ} \mathrm{C}$. The temperature of the column was programmed initially at $170^{\circ} \mathrm{C}$ for 8 minutes, then it was allowed to rise to $200^{\circ}$ $\mathrm{C}$ at a rate of $1^{0} \mathrm{C} / \mathrm{min}$ and the isothermal final period was 55 minutes. Thermal conductivity detectors were excellent. Nitrogen was used as a carrier gas at a flow rate of $11.4 \mathrm{ml} / \mathrm{min}$. Hydrogen flow was $10 \%$ above nitrogen flow. Standard fatty acid methyl esters were used for the identification of the sample fatty acid peaks. The following Standard fatty acids were used, the methyl esters of $\mathrm{C}_{8: 0} \mathrm{C}_{9: 0} \mathrm{C}_{10: 0} \mathrm{C}_{11: 0} \mathrm{C}_{12: 0} \mathrm{C}_{14: 0} \mathrm{C}$ ${ }_{16: 0} C_{18: 0} C_{18: 1} C_{18: 2} C_{18: 3} C_{20: 0} C_{22: 0}$. The peak area of each component was measured automatically by chromatograph machine. It was also measured by the actual physical measurement by the triangulation method (Yusuf et al, 1981). The total $\mathrm{mm}$ of all peak areas were taken as $100 \%$ and the percent population of a given fatty acid peak was calculated accordingly. The fatty acids were expressed as weight percentages of total fatty acids.

\section{Results and Discussion}

Vegetable oil is an important element in the diet of most transitional countries; nevertheless, little is known about the fatty acid composition of these oils. Therefore some seed oils are investigated here to check their fatty acid profiles and thereafter the oils were mixed to get effective $n-6 / n-3$ ratio.

Table 1. Saturated fatty acid profile of the investigated vegetable oil

\begin{tabular}{|l|c|c|c|c|c|}
\hline \multirow{2}{*}{ Oil or Fat } & \multicolumn{5}{|c|}{ Saturated fatty acid } \\
\cline { 2 - 6 } & $\begin{array}{c}\text { Arachic acid } \\
\text { C20:0 (\%) }\end{array}$ & $\begin{array}{c}\text { Lauric acid } \\
\text { C12:0 }(\%)\end{array}$ & $\begin{array}{c}\text { Myristic acid } \\
\text { C14:0 }(\%)\end{array}$ & $\begin{array}{c}\text { Palmitic acid } \\
\text { C16:0 }(\%)\end{array}$ & $\begin{array}{c}\text { Stearic acid } \\
\text { C18:0 }(\%)\end{array}$ \\
\hline Peanut & 5.0 & - & 4.0 & - & - \\
\hline Olive oil & - & - & - & - & - \\
\hline Linseed oil & - & - & - & 9.9 & 30.0 \\
\hline Sesame oil & - & - & - & - & 8.5 \\
\hline Soybean oil & - & - & - & 30.0 & - \\
\hline Sunflower oil & - & - & - & - & - \\
\hline
\end{tabular}

Table 1 shows the saturated fatty acid profile of the investigated vegetable oil. It was observed that highest proportion $(39.9 \%)$ saturated fatty acid contain linseed (tisi) oil after that second highest proportion (30\%) present in sunflower oil. There was no saturated fatty acid found in soybean oil and olive oil.

Table 2. Monounsaturated fatty acid profile of the investigated vegetable oil

\begin{tabular}{|l|c|c|c|c|}
\hline \multirow{2}{*}{ Oil or Fat } & \multicolumn{4}{|c|}{ Monounsaturated fatty acid } \\
\cline { 2 - 5 } & Palmitoleic acid & Oleic acid & Erucic acid & Palmitoleic acid \\
$\mathrm{C} 16: 1(\%)$ & $\mathrm{C} 18: 1(\%)$ & $\mathrm{C} 22: 1(\%)$ & - \\
\hline Peanut oil & - & - & - & - \\
\hline Olive oil & - & - & - & - \\
\hline Linseed oil & - & - & - & - \\
\hline Sesame oil & - & 37.1 & - & - \\
\hline Soybean oil & - & - & - & 3.0 \\
\hline Sunflower oil & 3.0 & - & - & - \\
\hline
\end{tabular}

Table 2 represents the monounsaturated fatty acid of the investigated vegetable oil. It was found that sesame (til) oil contains $37.1 \%$ oleic acid whereas sunflower oil contain 3\% palmitoleic and palmitoleic acid. Any types of monounsaturated fatty acid were not found in peanut, olive, linseed (tisi) and soybean oil. 
Table 3. Polyunsaturated fatty acid profile of the investigated vegetable oil

\begin{tabular}{|l|c|c|c|c|}
\hline \multirow{2}{*}{ Oil or Fat } & \multicolumn{4}{|c|}{ Polyunsaturated fatty acid } \\
\cline { 2 - 5 } & $\begin{array}{c}\text { Linoleic acid } \\
(\omega 6) \text { C18:2 }(\%)\end{array}$ & $\begin{array}{c}\text { Alpha Linolenic } \\
\text { acid }(\omega 3) \text { C18:3 }(\%)\end{array}$ & $\begin{array}{c}\text { Arachidonic acid } \\
(\omega 6) \text { C20:4 } \\
(\%)\end{array}$ & $\begin{array}{c}\text { Docosahexaenoic } \\
\text { acid }(\omega 3) \text { C22:6 (\%) }\end{array}$ \\
\hline Peanut oil & - & 75.0 & 10.0 & - \\
\hline Olive oil & 35.0 & 2.0 & 60.0 & - \\
\hline Linseed oil & 16.9 & 39.0 & - & - \\
\hline Sesame oil & 33.1 & 2.1 & 78.0 & - \\
\hline Soybean oil & - & 22.0 & 67.0 & - \\
\hline Sunflower oil & - & - & - & - \\
\hline
\end{tabular}

Table 3 shows polyunsaturated fatty acid profile of the investigated vegetable oils. In this study, it was observed that olive and soybean oil contain $100 \%$ polyunsaturated fatty acid. It was also found that olive oil contains higher proportion of not only arachidonic acid $(60 \%)$ but also linoleic $(35 \%)$ and docosahexaenoic acid (3\%). The beneficial health effects of olive oil are due to both its high content of polyunsaturated fatty acids and its high content of antioxidative substances. Studies have shown that olive oil offers protection against heart disease by controlling LDL-cholesterol levels while raising HDL levels (Keys et al., 1986). The soybean is the world's leading source of edible oil. Soybean oil is a good source of both linoleic and linolenic acids, which are essential for humans. More than $50 \%$ of the fat in soybean oil is linoleic acid, while about $7 \%$ of the total fat is linolenic. Soybean oil contains only $22 \%$ alpha linolenic and $78 \%$ arachidonic acid. Peanut oil contains $75 \%$ alpha linolenic acid and $10 \%$ arachidonic acid. The lowest percentages $(35.2 \%)$ of polyunsaturated fatty acid were found in sesame (til) oil.

It is possible to maintain healthy ratios of $n-6: n-3$ range from $1: 1$ to $4: 1$ if we mixed vegetable oils by following proportion: sunflower oil $(66.7 \%)$ and linseed (tisi) oil $(33.3 \%)$, sunflower oil $(81.3 \%)$ and peanut oil $(18.7 \%)$, soybean oil $(87 \%)$ and olive oil $(13 \%)$ soybean oil $(66.7 \%)$ and sesame (til) oil (33.3\%) soybean oil $(87 \%)$ and sunflower oil $(13 \%)$. The author investigated the above mixed oils and found that sunflower oil $(66.7 \%)$ and linseed (tisi) oil (33.3\%) contains n-6/n-3 fatty acid ratio $=3.9$. Sunflower oil $(81.3 \%)$ and peanut oil $(18.7 \%)$ contains $n-6 / n-3$ fatty acid ratio $=4.01$. Soybean oil $(87 \%)$ and olive oil $(13 \%)$ contain $n-6 / n-3$ fatty acid ratio $=4.05$. Soybean oil $(66.7 \%)$ and sesame (til) oil $(33.3 \%)$ contain $n-6 / n-3$ fatty acid ratio $=4.10$. Soybean oil $(87 \%)$ and sunflower oil $(13 \%)$ contain $n-6 / n-3$ fatty acid ratio $=4.0$ (Table 4$)$.

Table 4. Mixed vegetable oils with effective $n-6 / n-3$ fatty acid ratio

\begin{tabular}{|l|c|c|}
\hline \multicolumn{1}{|c|}{ Vegetable Mixed } & Proportion & n-6/n-3 fatty acid ratio \\
\hline Sunflower oil + Linseed oil & $66.7 \%+33.3 \%$ & 3.9 \\
\hline Sunflower oil + Peanut oil & $81.3 \%+18.7 \%$ & 4.01 \\
\hline Soybean oil + Olive oil & $87 \%+13 \%$ & 4.05 \\
\hline Soybean oil + Sesame oil & $66.7 \%+33.3 \%$ & 4.10 \\
\hline Soybean oil + Sunflower oil & $87 \%+13 \%$ & 4.0 \\
\hline
\end{tabular}

\section{Conclusions}

Sesame and olive oil have been supposed to possess the highest position in nutritional quality while compared to peanut or sunflower by the fatty acid composition. Soybean oil contains the balanced omega-6/omega-3 fatty acid ratio. Both olive and peanut oil could be consumed as vegetable oils like the soybean oil. Sunflower with peanut, linseed and soybean oil contained the effective ratio of n-6/n-3 fatty acids which may reduce the mortality from cardiovascular disease, suppressed inflammation in patients with rheumatoid arthritis and may decrease risk of breast cancer.

\section{References}

Binkoski, A. E.; Kris-Etherton, P. M. and Wilson, T. A. 2005. Balance of unsaturated fatty acids is important to a cholesterol-lowering diet: Comparison of midoleic sunflower oil and olive oil on cardiovascular disease risk factors. J. Am. Diet. Assoc., 105: 10801086.

Bucher, H. C.; Peter, H.; Christian, S. and Meier, G. 2002. $n-3$ polyunsaturated fatty acids in coronary heart disease: a meta-analysis of randomized 
controlled trials. The American Journal of Medicine, 112(4): 298-304.

Harris, W. S.; Poston, W. C. and Haddock, C. K. 2007. Tissue n-3 and n-6 fatty acids and risk for coronary heart disease events. Atherosclerosis, 193: 1-10.

Keys, A.; Menotti, A.; Karvonen, M. J.; Aravanis, C.; Blackburn, H.; Buzina, R.; Djordjevic, B. S.; Dontas, A. S.; Fidanza, F. and Keys, M. H. 1986. The diet and 15-year death rate in the seven countries study. Am. J. Epidemiol., 124: 903-15.

Okuyama, H. 2001. High $n-6$ to $n-3$ ratio of dietary fatty acids rather than serum cholesterol as a major risk factor for coronary heart disease. European Journal Lipid Science Technology, 103: 418-422.

Riediger, N. D.; Azordegan, N.; Harris-Janz, S.; Ma, D. W.; Suh, M. and Moghadasian, M. H. 2009. Designer oils low in n-6:n-3 fatty acid ratio beneficially modifies cardiovascular risks in mice. European Journal Nutrition, 31: 120-25.

Simopoulos, A. P. 2008. The importance of the omega6/omega-3 fatty acid ratio in cardiovascular disease and other chronic diseases. Exp. Biological Medicine (Maywood), 233(6): 674-88.

Tri6bole, E. F. 2006. Excess Omega-6 Fats Thwart Health Benefits from Omega-3 Fats. British Medical Journal, 332: 752-56

USDA (United States Department of Agriculture). 1997. Composition of Foods: Fats and Oils. Washington, DC: USDA.

Wijendran, V. and Hayes, K. C. 2004. Dietary n-6 and n-3 fatty acid balance and cardiovascular health, Annual Review Nutrition, 24: 597-615.

Yusuf, H.; Alim, K. M.; Rahman, R.; Quazi, S. and Hossain, A. 1993. Fatty acids of 12 marine fish species of the Bay of Bengal. J. Fd. comp. Annual, 6: 346-353. 